IJMMS 25:8 (2001) 517-524

PII. S0161171201003982

http://ijmms.hindawi.com

(C) Hindawi Publishing Corp.

\title{
TWO-WEIGHT NORM INEQUALITIES FOR THE ROUGH FRACTIONAL INTEGRALS
}

\author{
YONG DING and CHIN-CHENG LIN
}

(Received 1 October 1999)

\begin{abstract}
The authors give the weighted $\left(L^{p}, L^{q}\right)$-boundedness of the rough fractional integral operator $T_{\Omega, \alpha}$ and the fractional maximal operator $M_{\Omega, \alpha}$ with two different weight functions.
\end{abstract}

2000 Mathematics Subject Classification. Primary 42B20, 42B25.

1. Introduction. Suppose that $0<\alpha<n, \Omega(x)$ is homogeneous of degree zero on $\mathbb{R}^{n}$ and $\Omega\left(x^{\prime}\right) \in L^{s}\left(S^{n-1}\right)(s>1)$, where $S^{n-1}$ denotes the unit sphere in $\mathbb{R}^{n}$. Then the fractional integral operator $T_{\Omega, \alpha}$ and the maximal operator $M_{\Omega, \alpha}$ are defined by

$$
\begin{gathered}
T_{\Omega, \alpha} f(x)=\int_{\mathbb{R}^{n}} \frac{\Omega(x-y)}{|x-y|^{n-\alpha}} f(y) d y, \\
M_{\Omega, \alpha} f(x)=\sup _{r>0} \frac{1}{r^{n-\alpha}} \int_{|x-y|<r}|\Omega(x-y)||f(y)| d y,
\end{gathered}
$$

respectively. It is easy to see that, when $\Omega \equiv 1, T_{\Omega, \alpha}$ and $M_{\Omega, \alpha}$ are the usual fractional integral operator $I_{\alpha}$ and the maximal operator $M_{\alpha}$. In 1971, Muckenhoupt and Wheeden [6] gave $\left(L^{p}, L^{q}\right)$-boundedness with power weight of $T_{\Omega, \alpha}$. In 1993, Chanillo, Watson and Wheeden [1] proved that when $s \geq n /(n-\alpha)$, the operator $T_{\Omega, \alpha}$ is weak type $(1, n /(n-\alpha))$. Recently, we gave the weighted $\left(L^{p}, L^{q}\right)$-boundedness of $T_{\Omega, \alpha}$ and $M_{\Omega, \alpha}$ for general $A(p, q)$ weight [3], and the weak boundedness of $T_{\Omega, \alpha}$ and $M_{\Omega, \alpha}$ with power weight [2].

The purpose of this paper is to study the weighted $\left(L^{p}, L^{q}\right)$-boundedness of $T_{\Omega, \alpha}$ and $M_{\Omega, \alpha}$ for the two different weights.

Before showing our results, we give the definitions of some weight classes. In the following definitions, the function $\omega$ and the function pair $(u, v)$ are all locally integrable nonnegative functions. Moreover, $C>0$ and $Q$ denotes a cube in $\mathbb{R}^{n}$ with its sides parallel to the coordinate axes and $\chi_{Q}(x)$ denotes the characterization function of $Q$.

The Definition of $A_{p}(1<p<\infty)$. A function $\omega$ is said to belong to $A_{p}(1<$ $p<\infty)$ if

$$
\sup _{Q \subset \mathbb{R}^{n}}\left(\frac{1}{|Q|} \int_{Q} \omega(x) d x\right)\left(\frac{1}{|Q|} \int_{Q} \omega(x)^{-1 /(p-1)} d x\right)^{p-1} \leq C .
$$


The Definition of $A(p, q)(1<p, q<\infty)$. A function $\omega$ is said to belong to $A(p, q)(1<p, q<\infty)$ if

$$
\sup _{Q \subset \mathbb{R}^{n}}\left(\frac{1}{|Q|} \int_{Q} \omega(x)^{q} d x\right)^{1 / q}\left(\frac{1}{|Q|} \int_{Q} \omega(x)^{-p^{\prime}} d x\right)^{1 / p^{\prime}} \leq C .
$$

THE DEFINITION OF $A_{p}^{*}(1<p<\infty)$. A function pair $(u, v)$ is said to belong to $A_{p}^{*}(1<p<\infty)$ if

$$
\sup _{Q \subset \mathbb{R}^{n}}\left(\frac{1}{|Q|} \int_{Q} u(x) d x\right)\left(\frac{1}{|Q|} \int_{Q} v(x)^{-1 /(p-1)} d x\right)^{p-1} \leq C .
$$

THE Definition of $A^{*}(p, q)(1<p, q<\infty)$. A function pair $(u, v)$ is said to belong to $A^{*}(p, q)(1<p, q<\infty)$ if

$$
\sup _{Q \subset \mathbb{R}^{n}}\left(\frac{1}{|Q|} \int_{Q} u(x)^{q} d x\right)^{1 / q}\left(\frac{1}{|Q|} \int_{Q} v(x)^{-p^{\prime}} d x\right)^{1 / p^{\prime}} \leq C .
$$

The Definition of $S_{p}^{*}(1<p<\infty)$. A function pair $(u, v)$ is said to belong to $S_{p}^{*}(1<p<\infty)$ if

$$
\int_{Q}\left[M\left(v^{-1 /(p-1)} \chi_{Q}\right)\right]^{p} u(x) d x \leq C \int_{Q} v(x)^{-1 /(p-1)} d x .
$$

In this paper, we prove the following results.

THEOREM 1.1. Suppose that $0<\alpha<n, 1<p<n / \alpha, 1 / q=1 / p-\alpha / n, \Omega$ is homogeneous of degree zero defined on $\mathbb{R}^{n}$ and $\Omega \in L^{s}\left(S^{n-1}\right)$. If $p, q, s$, and $(u, v)$ satisfy one of the following conditions:

(a) $1 \leq s^{\prime}<p,\left(u^{s^{\prime}}, v^{s^{\prime}}\right) \in A^{*}\left(p / s^{\prime}, q / s^{\prime}\right)$, in addition $u(x)^{s^{\prime}}, v(x)^{s^{\prime}} \in A\left(p / s^{\prime}, q / s^{\prime}\right)$;

(b) $s>q,\left(v^{-s^{\prime}}, u^{-s^{\prime}}\right) \in A^{*}\left(q^{\prime} / s^{\prime}, p^{\prime} / s^{\prime}\right)$, in addition $v(x)^{-s^{\prime}}, u(x)^{-s^{\prime}} \in A\left(q^{\prime} / s^{\prime}, p^{\prime} / s^{\prime}\right)$; then there is a constant $C$, independent of $f$, such that $T_{\Omega, \alpha}$ satisfies

$$
\left(\int_{\mathbb{R}^{n}}\left|T_{\Omega, \alpha} f(x) u(x)\right|^{q} d x\right)^{1 / q} \leq C\left(\int_{\mathbb{R}^{n}}|f(x) v(x)|^{p} d x\right)^{1 / p} .
$$

THEOREM 1.2. Suppose that $0<\alpha<n, 1<p<n / \alpha, 1 / q=1 / p-\alpha / n$, $\Omega$ is homogeneous of degree zero defined on $\mathbb{R}^{n}$ and $\Omega \in L^{s}\left(S^{n-1}\right)$. If $p, q, s$, and $(u, v)$ satisfy one of the following conditions:

(c) $1 \leq s^{\prime}<p$, $\left(u^{s^{\prime}}, v^{s^{\prime}}\right) \in A^{*}\left(p / s^{\prime}, q / s^{\prime}\right)$, in addition $\sigma=v^{-s^{\prime}\left(p / s^{\prime}\right)^{\prime}}$ satisfies the doubling condition;

(d) $s>q,\left(v^{-s^{\prime}}, u^{-s^{\prime}}\right) \in A^{*}\left(q^{\prime} / s^{\prime}, p^{\prime} / s^{\prime}\right)$, in addition $v(x)^{-s^{\prime}}, u(x)^{-s^{\prime}} \in A\left(q^{\prime} / s^{\prime}\right.$, $\left.p^{\prime} / s^{\prime}\right)$;

then there is a constant $C$, independent of $f$, such that $M_{\Omega, \alpha}$ satisfies

$$
\left(\int_{\mathbb{R}^{n}}\left[M_{\Omega, \alpha} f(x) u(x)\right]^{q} d x\right)^{1 / q} \leq C\left(\int_{\mathbb{R}^{n}}|f(x) v(x)|^{p} d x\right)^{1 / p} .
$$

2. Some elementary properties of the weight class. We begin by giving some properties of the weight classes $A_{p}, A(p, q), A_{p}^{*}, A^{*}(p, q)$, and $S_{p}^{*}$. 
THE ELEMENTARY PROPERTIES OF $A_{p}(1<p<\infty)$

(a) $A_{p_{1}} \subset A_{p_{2}}$, if $1<p_{1}<p_{2}<\infty$.

(b) $\omega(x) \in A_{p}$, if and only if $\omega(x)^{1-p^{\prime}} \in A_{p^{\prime}}$.

(c) If $\omega(x) \in A_{p}$, then there is an $\varepsilon>0$ such that $p-\varepsilon>1$ and $\omega(x) \in A_{p-\varepsilon}$.

(d) If $\omega(x) \in A_{p}$, then there is an $\varepsilon>0$ such that $\omega(x)^{1+\varepsilon} \in A_{p}$.

(e) If $\omega(x) \in A_{p}$, then for any $0<\varepsilon<1, \omega(x)^{\varepsilon} \in A_{p}$.

(f) If $\omega(x) \in A_{p}$, then there are $C>0$ and $\varepsilon>0$ such that, for any $Q \in \mathbb{R}^{n}$,

$$
\frac{1}{|Q|} \int_{Q} \omega(x)^{1+\varepsilon} d x \leq C\left(\int_{Q} \omega(x) d x\right)^{1+\varepsilon} .
$$

See [4] for the proof.

THE ELEMENTARY PROPERTIES OF $A_{p}^{*}(1<p<\infty)$

(i) $A_{p_{1}}^{*} \subset A_{p_{2}}^{*}$, if $1<p_{1}<p_{2}<\infty$.

(ii) $(u, v) \in A_{p}^{*}$, if and only if $\left(v^{1-p^{\prime}}, u^{1-p^{\prime}}\right) \in A_{p^{\prime}}^{*}$.

(iii) $S_{p}^{*} \subset A_{p}^{*}$, for $1<p<\infty$.

(iv) If $(u, v) \in A_{p}^{*}$, then for any $0<\varepsilon<1,\left(u^{\varepsilon}, v^{\varepsilon}\right) \in S_{p}^{*}$.

(v) If $(u, v) \in A_{p}^{*}$ and $u(x), v(x) \in A_{p}$, then $(u, v) \in S_{p}^{*}$ and $\left(v^{1-p^{\prime}}, u^{1-p^{\prime}}\right) \in S_{p^{\prime}}^{*}$.

(vi) If $(u, v) \in A_{p}^{*}$ and $u(x), v(x) \in A_{p}$, then there is an $\varepsilon>0$ such that $\left(u^{1+\varepsilon}, v^{1+\varepsilon}\right) \in$ $A_{p}^{*}$ and $\left(v^{\left(1-p^{\prime}\right)(1+\varepsilon)}, u^{\left(1-p^{\prime}\right)(1+\varepsilon)}\right) \in A_{p^{\prime}}^{*}$.

(vii) If $(u, v) \in A_{p}^{*}$ and $u(x), v(x) \in A_{p}$, then there is an $\varepsilon>0$ such that $p-\varepsilon>1$ and $(u, v) \in A_{p-\varepsilon}^{*}$.

Proof. The proof of (i) and (ii) can be deduced from the definition of $A_{p}^{*}$.

For (iii), by [8], we know that the Hardy-Littlewood maximal operator $M$ is bounded from $L^{p}(v)$ to $L^{p}(u)(1<p<\infty)$ if and only if $(u, v) \in S_{p}^{*}$. On the other hand, by [5], the operator $M$ is bounded from $L^{p}(v)$ to weak $L^{p}(u)$ if and only if $(u, v) \in A_{p}^{*}$. Hence, (iii) holds.

The proof of (iv) is a conclusion in [7].

Now, we prove (v). Since $v(x) \in A_{p}$, we have $v(x)^{1-p^{\prime}} \in A_{p^{\prime}}$, by (b). Thus, from (f) there are $C>0$ and $\eta>0$ such that, for any $Q \in \mathbb{R}^{n}$,

$$
\begin{gathered}
\frac{1}{|Q|} \int_{Q} u(x)^{1+\eta} d x \leq C\left(\int_{Q} u(x) d x\right)^{1+\eta}, \\
\frac{1}{|Q|} \int_{Q} v(x)^{\left(1-p^{\prime}\right)(1+\eta)} d x \leq C\left(\int_{Q} v(x)^{1-p^{\prime}} d x\right)^{1+\eta},
\end{gathered}
$$

that is,

$$
\frac{1}{|Q|} \int_{Q} v(x)^{-(1+\eta) /(p-1)} d x \leq C\left(\int_{Q} v(x)^{-1 /(p-1)} d x\right)^{1+\eta} .
$$

Hence, by $(u, v) \in A_{p}^{*},(2.2)$, and (2.4) we have

$$
\begin{aligned}
& \sup _{Q}\left(\frac{1}{|Q|} \int_{Q} u(x)^{1+\eta} d x\right)\left(\frac{1}{|Q|} \int_{Q} v(x)^{-(1+\eta) /(p-1)} d x\right)^{p-1} \\
& \quad \leq C\left(\sup _{Q}\left(\frac{1}{|Q|} \int_{Q} u(x) d x\right)\left(\frac{1}{|Q|} \int_{Q} v(x)^{-1 /(p-1)} d x\right)^{p-1}\right)^{1+\eta}<\infty,
\end{aligned}
$$


that is,

$$
\left(u^{1+\eta}, v^{1+\eta}\right) \in A_{p}^{*} .
$$

Taking $\delta=1 /(1+\eta)$, then $0<\delta<1$ and $(u, v)=\left(u^{(1+\eta) \delta}, v^{(1+\eta) \delta}\right) \in S_{p}^{*}$ by $(2.6)$ and (iv). On the other hand, by (2.6) and (ii) we get $\left(v^{(1+\eta)\left(1-p^{\prime}\right)}, u^{(1+\eta)\left(1-p^{\prime}\right)}\right) \in A_{p^{\prime}}^{*}$. As above, we take $\delta=1 /(1+\eta)$, then $\left(v^{1-p^{\prime}}, u^{1-p^{\prime}}\right) \in S_{p^{\prime}}^{*}$. This is (v).

By (2.6), we have $\left(u^{1+\eta}, v^{1+\eta}\right) \in A_{p}^{*}$. Now, we take $0<\delta<1$ such that $\delta(1+\eta)>1$. Let $1+\varepsilon=\delta(1+\eta)$, then $\varepsilon>0$ and

$$
\left(u^{1+\varepsilon}, v^{1+\varepsilon}\right)=\left(u^{\delta(1+\eta)}, v^{\delta(1+\eta)}\right) \in S_{p}^{*} \subset A_{p}^{*},
$$

by (iii) and (iv). From (2.7) and (ii), we can get $\left(v^{\left(1-p^{\prime}\right)(1+\varepsilon)}, u^{\left(1-p^{\prime}\right)(1+\varepsilon)}\right) \in A_{p^{\prime}}^{*}$. Thus, we prove (vi). Finally, we prove (vii). By (vi), there is an $\eta>0$ such that

$$
\left(v^{\left(1-p^{\prime}\right)(1+\eta)}, u^{\left(1-p^{\prime}\right)(1+\eta)}\right) \in A_{p^{\prime}}^{*} .
$$

Taking $\varepsilon=\eta(p-1) /(1+\eta)$, then we can see easily that $\varepsilon>0$ and $1<p-\varepsilon<p$. Hence, we have $p^{\prime}<(p-\varepsilon)^{\prime}$ and $\left(v^{\left(1-p^{\prime}\right)(1+\eta)}, u^{\left(1-p^{\prime}\right)(1+\eta)}\right) \in A_{(p-\varepsilon)^{\prime}}^{*}$, by (i). From (ii), we get $\left(u^{\left(1-p^{\prime}\right)(1+\eta)[1-(p-\varepsilon)]}, v^{\left(1-p^{\prime}\right)(1+\eta)[1-(p-\varepsilon)]}\right) \in A_{p-\varepsilon}^{*}$. However, $\left(1-p^{\prime}\right)(1+\eta)[1-$ $(p-\varepsilon)]=1$. Thus, we have $(u, v) \in A_{p-\varepsilon}^{*}$.

THE RELATIONS BETWEen $A_{p}$ AND $A(p, q), A_{p}^{*}$, AND $A^{*}(p, q)$. Suppose that $0<\alpha<n, 1<p<n / \alpha, 1 / q=1 / p-\alpha / n$, then we have the following conclusions:

$$
\begin{aligned}
& \omega(x) \in A(p, q) \Leftrightarrow \omega(x)^{q} \in A_{q(n-\alpha) / n} \Longleftrightarrow \omega(x)^{-p^{\prime}} \in A_{1+p^{\prime} / q}, \\
&(u, v) \in A^{*}(p, q) \Longleftrightarrow\left(u^{q}, v^{q}\right) \in A_{q(n-\alpha) / n}^{*} \Longleftrightarrow\left(v^{-p^{\prime}}, u^{-p^{\prime}}\right) \in A_{1+p^{\prime} / q}^{*} .
\end{aligned}
$$

Equations (2.9) and (2.10) can be deduced from the definitions of $A_{p}$ and $A(p, q)$, $A_{p}^{*}$ and $A^{*}(p, q)$, respectively. Here we omit the details.

3. Proofs of the theorems. The proofs of the theorems are based on Wheeden's a result in [9] and some lemmas.

THEOREM 3.1 (see [9]). For $0<\alpha<n, 1<p<n / \alpha$, and $1 / q=1 / p-\alpha / n, M_{\alpha}$ is bounded from $L^{p}\left(v^{p}\right)$ to $L^{q}\left(u^{q}\right)$ if and only if the weights pair $(u, v) \in A^{*}(p, q)$ and $\sigma=v^{-p^{\prime}}$ satisfies the doubling condition. That is, there is a constant $C>0$ such that $\sigma(2 B) \leq C \sigma(B)$ for all ball $B$ in $\mathbb{R}^{n}$.

The following lemma gives a pointwise relation between $T_{\Omega, \alpha}$ and $M_{\Omega, \alpha}$.

LEMMA 3.2 (see [3]). For any $\varepsilon>0$ with $0<\alpha-\varepsilon<\alpha+\varepsilon<n$, we have

$$
\left|T_{\Omega, \alpha} f(x)\right| \leq C\left[M_{\Omega, \alpha+\varepsilon} f(x)\right]^{1 / 2} \cdot\left[M_{\Omega, \alpha-\varepsilon} f(x)\right]^{1 / 2}, \quad x \in \mathbb{R}^{n},
$$

where C depends only on $\alpha, \varepsilon, n$.

The following two lemmas characterize an important property of $A^{*}(p, q)$ weights.

LEMmA 3.3. Suppose that $0<\alpha<n, 1<p<n / \alpha, 1 / q=1 / p-\alpha / n,(u, v) \in$ $A^{*}(p, q)$, and $u(x), v(x) \in A(p, q)$. Then there is an $\varepsilon>0$ such that $\varepsilon<\alpha<\alpha+\varepsilon<$ $n ; 1 / p>(\alpha+\varepsilon) / n, 1 / q<(n-\varepsilon) / n$, and $(u, v) \in A^{*}\left(p, q_{\varepsilon}\right),(u, v) \in A^{*}\left(p, \bar{q}_{\varepsilon}\right)$ hold at the same time, where $1 / q_{\varepsilon}=1 / p-(\alpha+\varepsilon) / n, 1 / \bar{q}_{\varepsilon}=1 / p-(\alpha-\varepsilon) / n$. 
Proof. For $\alpha>0,1 / q<1$, we may take $\delta_{1}>0$ such that $\delta_{1}<\alpha$ and $1 / q+\delta_{1} / n<1$. Let

$$
\frac{1}{q_{\delta_{1}}}=\frac{1}{p}-\frac{\alpha-\delta_{1}}{n}=\frac{1}{q}+\frac{\delta_{1}}{n},
$$

then $q>q_{\delta_{1}}>1$ and $1+p^{\prime} / q<1+p^{\prime} / q_{\delta_{1}}$. By $(u, v) \in A^{*}(p, q),(2.10)$, and (i), we have

$$
\left(v^{-p^{\prime}}, u^{-p^{\prime}}\right) \in A_{1+p^{\prime} / q}^{*} \subset A_{1+p^{\prime} / q_{\delta_{1}}}^{*} .
$$

Since $0<\alpha-\delta_{1}<n, 1<p<n /\left(\alpha-\delta_{1}\right)$, then by (3.2) and (2.10), we know that (3.3) is equivalent to

$$
(u, v) \in A^{*}\left(p, q_{\delta_{1}}\right) .
$$

On the other hand, by $(u, v) \in A^{*}(p, q)$ and $u(x), v(x) \in A(p, q)$, we get

$$
\left(v^{-p^{\prime}}, u^{-p^{\prime}}\right) \in A_{1+p^{\prime} / q}^{*}, \quad v(x)^{-p^{\prime}}, u(x)^{-p^{\prime}} \in A_{1+p^{\prime} / q},
$$

by (2.10) and (2.9), respectively. From (3.5) and (vii), we know that there is an $\eta$ satisfying $0<\eta<1 / q$ such that

$$
\left(v^{-p^{\prime}}, u^{-p^{\prime}}\right) \in A_{1+p^{\prime}(1 / q-\eta)}^{*} .
$$

Obviously, we can also choose $\delta_{2}>0$ small enough such that $\delta_{2}<\min \{\alpha, n-\alpha\}, 1 / p>$ $\left(\alpha+\delta_{2}\right) / n$, and $\delta_{2} / n<\eta$ hold at the same time. Now, let $1 / q_{\delta_{2}}=1 / p-\left(\alpha+\delta_{2}\right) / n$, then by $1 / p>\left(\alpha+\delta_{2}\right) / n$ and $\delta_{2} / n<\eta$, we get $0<1 / q_{\delta_{2}}<1$ and $1 / q_{\delta_{2}}=1 / q-\delta_{2} / n>$ $1 / q-\eta$. From this and (3.6), we have

$$
\left(v^{-p^{\prime}}, u^{-p^{\prime}}\right) \in A_{1+p^{\prime}(1 / q-\eta)}^{*} \subset A_{1+p^{\prime} / q_{\delta_{2}}}^{*} .
$$

Since $0<\alpha+\delta_{2}<n, 1<p<n /\left(\alpha+\delta_{2}\right)$, and $1 / q_{\delta_{2}}=1 / p-\left(\alpha+\delta_{2}\right) / n$, then by (2.10) we know that (3.7) is equivalent to

$$
(u, v) \in A^{*}\left(p, q_{\delta_{2}}\right) .
$$

Finally, let $\varepsilon=\min \left\{\delta_{1}, \delta_{2}\right\}$ and $1 / q_{\varepsilon}=1 / p-(\alpha+\varepsilon) / n, 1 / \bar{q}_{\varepsilon}=1 / p-(\alpha-\varepsilon) / n$. Then by (3.4) and (3.8), we get $(u, v) \in A^{*}\left(p, q_{\varepsilon}\right)$ and $(u, v) \in A^{*}\left(p, \bar{q}_{\varepsilon}\right)$. Thus, the proof of Lemma 3.3 is complete.

LEMmA 3.4. Suppose that $0<\alpha<n, 1 \leq s^{\prime}<p<n / \alpha, 1 / q=1 / p-\alpha / n$ and $\left(u^{s^{\prime}}, v^{s^{\prime}}\right) \in A^{*}\left(p / s^{\prime}, q / s^{\prime}\right)$. Moreover, $u(x)^{s^{\prime}}, v(x)^{s^{\prime}} \in A\left(p / s^{\prime}, q / s^{\prime}\right)$. Then there is an $\varepsilon>0$ such that

$$
\begin{gathered}
\varepsilon<\alpha<\alpha+\varepsilon<n ; \\
\frac{1}{p}>\frac{\alpha+\varepsilon}{n}, \quad \frac{1}{q}<\frac{n-\varepsilon}{n},
\end{gathered}
$$

and $\left(u^{s^{\prime}}, v^{s^{\prime}}\right) \in A^{*}\left(p / s^{\prime}, q_{\varepsilon} / s^{\prime}\right),\left(u^{s^{\prime}}, v^{s^{\prime}}\right) \in A^{*}\left(p / s^{\prime}, \bar{q}_{\varepsilon} / s^{\prime}\right)$ hold at the same time. Where $1 / q_{\varepsilon}=1 / p-(\alpha+\varepsilon) / n$ and $1 / \bar{q}_{\varepsilon}=1 / p-(\alpha-\varepsilon) / n$. 
Proof. Since $1 /\left(q / s^{\prime}\right)=1 /\left(p / s^{\prime}\right)-\alpha s^{\prime} / n$, from Lemma 3.3, there is an $\eta>0$ such that $\eta<\alpha s^{\prime}<\alpha s^{\prime}+\eta<n, 1 /\left(p / s^{\prime}\right)>\left(\alpha s^{\prime}+\eta\right) / n, 1 /\left(q / s^{\prime}\right)<(n-\eta) / n$ and

$$
\left(u^{s^{\prime}}, v^{s^{\prime}}\right) \in A^{*}\left(\frac{p}{s^{\prime}}, q_{\eta}\right), \quad\left(u^{s^{\prime}}, v^{s^{\prime}}\right) \in A^{*}\left(\frac{p}{s^{\prime}}, \bar{q}_{\eta}\right)
$$

hold at the same time, where

$$
\frac{1}{q_{\eta}}=\frac{1}{p / s^{\prime}}-\frac{\alpha s^{\prime}+\eta}{n}, \quad \frac{1}{\bar{q}_{\eta}}=\frac{1}{p / s^{\prime}}-\frac{\alpha s^{\prime}-\eta}{n} .
$$

Let $\varepsilon=\eta / s^{\prime}, q_{\varepsilon}=s^{\prime} q_{\eta}$, and $\bar{q}_{\varepsilon}=s^{\prime} \bar{q}_{\eta}$, then it is easy to see that $\varepsilon$ satisfies (3.9) and (3.10). Moreover, by (3.11) and (3.12) we know that

$$
\left(u^{s^{\prime}}, v^{s^{\prime}}\right) \in A^{*}\left(\frac{p}{s^{\prime}}, \frac{q_{\varepsilon}}{s^{\prime}}\right), \quad\left(u^{s^{\prime}}, v^{s^{\prime}}\right) \in A^{*}\left(\frac{p}{s^{\prime}}, \frac{\bar{q}_{\varepsilon}}{s^{\prime}}\right),
$$

hold at the same time, where $1 / q_{\varepsilon}=1 / p-(\alpha+\varepsilon) / n$ and $1 / \bar{q}_{\varepsilon}=1 / p-(\alpha-\varepsilon) / n$. This completes the proof of Lemma 3.4.

Now, we turn to the proofs of Theorems 1.1 and 1.2. We alternatively prove them.

THE PROOF OF (1.8) FOR THE CONDITION (c) IN THEOREM 1.2. Note that, for $r>0$, $\left(\int_{|x-y|<r}|\Omega(x-y)|^{s} d y\right)^{1 / s} \leq C r^{n / s}\|\Omega\|_{L^{s}\left(S^{n-1}\right)}$. Hence, we have

$$
M_{\Omega, \alpha} f(x) \leq C\left[M_{\alpha s^{\prime}}\left(|f|^{s^{\prime}}\right)(x)\right]^{1 / s^{\prime}} .
$$

Since $1 \leq s^{\prime}<p<n / \alpha$ and $1 / q=1 / p-\alpha / n$, we get $0<\alpha s^{\prime}<n, 1<p / s^{\prime}<$ $n / \alpha s^{\prime}$, and $1 /\left(q / s^{\prime}\right)=1 /\left(p / s^{\prime}\right)-\alpha s^{\prime} / n$. Moreover, $\left(u^{s^{\prime}}, v^{s^{\prime}}\right) \in A^{*}\left(p / s^{\prime}, q / s^{\prime}\right)$ and $\sigma=\left(v^{s^{\prime}}\right)^{-\left(p / s^{\prime}\right)^{\prime}}$ satisfies the doubling condition by (c). From Theorem 3.1, we know that the operator $M_{\alpha s^{\prime}}$ is bounded from $L^{p / s^{\prime}}\left(\left(v^{s^{\prime}}\right)^{p / s^{\prime}}\right)$ to $L^{q / s^{\prime}}\left(\left(u^{s^{\prime}}\right)^{q / s^{\prime}}\right)$. Thus, we get

$$
\begin{aligned}
\left(\int_{\mathbb{R}^{n}}\left[M_{\Omega, \alpha} f(x) u(x)\right]^{q} d x\right)^{1 / q} & \leq C\left(\int_{\mathbb{R}^{n}}\left[M_{\alpha s^{\prime}}\left(|f|^{s^{\prime}}\right)(x) u(x)^{s^{\prime}}\right]^{q / s^{\prime}} d x\right)^{1 / q} \\
& \leq C\left(\int_{\mathbb{R}^{n}}|f(x)|^{p} v(x)^{p} d x\right)^{1 / p} .
\end{aligned}
$$

This completes the proof of (1.8) for the condition (c).

THE PROOF OF (1.7) FOR THE CONDITION (a) IN THEOREM 1.1. First, we show that, under the condition (a) in Theorem 1.1, $\sigma=v^{-s^{\prime}\left(p / s^{\prime}\right)^{\prime}}$ still satisfies the doubling condition. In fact, by $v(x)^{s^{\prime}} \in A\left(p / s^{\prime}, q / s^{\prime}\right)$ and (2.9), we have $\left(v(x)^{s^{\prime}}\right)^{-\left(p / s^{\prime}\right)^{\prime}} \in$ $A_{1+\left(p / s^{\prime}\right)^{\prime} /\left(q / s^{\prime}\right)} \subset A_{\infty}$. Since, every weight function in $A_{\infty}$ satisfies the doubling condition, and so does $\sigma=v^{-s^{\prime}\left(p / s^{\prime}\right)^{\prime}}$.

By Lemma 3.4, there is an $\varepsilon>0$ satisfying (3.9) and (3.10) such that

$$
\left(u^{s^{\prime}}, v^{s^{\prime}}\right) \in A^{*}\left(\frac{p}{s^{\prime}}, \frac{q_{\varepsilon}}{s^{\prime}}\right), \quad\left(u^{s^{\prime}}, v^{s^{\prime}}\right) \in A^{*}\left(\frac{p}{s^{\prime}}, \frac{\bar{q}_{\varepsilon}}{s^{\prime}}\right)
$$

hold at the same time. Where $1 / q_{\varepsilon}=1 / p-(\alpha+\varepsilon) / n$ and $1 / \bar{q}_{\varepsilon}=1 / p-(\alpha-\varepsilon) / n$. Let $l_{1}=2 q_{\varepsilon} / q, l_{2}=2 \bar{q}_{\varepsilon} / q$, then $1 / l_{1}+1 / l_{2}=1$. For given $\varepsilon>0$ above, using (3.1) and 
Hölder's inequality, we have

$$
\begin{aligned}
\left\|T_{\Omega, \alpha} f\right\|_{q, u^{q}} & \leq C\left(\int_{\mathbb{R}^{n}}\left[M_{\Omega, \alpha+\varepsilon} f(x) u(x)\right]^{q l_{1} / 2} d x\right)^{1 / q l_{1}}\left(\int_{\mathbb{R}^{n}}\left[M_{\Omega, \alpha-\varepsilon} f(x) u(x)\right]^{q l_{2} / 2} d x\right)^{1 / q l_{2}} \\
& =C\left(\int_{\mathbb{R}^{n}}\left[M_{\Omega, \alpha+\varepsilon} f(x) u(x)\right]^{q_{\varepsilon}} d x\right)^{1 / 2 q_{\varepsilon}}\left(\int_{\mathbb{R}^{n}}\left[M_{\Omega, \alpha-\varepsilon} f(x) u(x)\right]^{\bar{q}_{\varepsilon}} d x\right)^{1 / 2 \bar{q}_{\varepsilon}} .
\end{aligned}
$$

Note that $\sigma=v^{-s^{\prime}\left(p / s^{\prime}\right)^{\prime}}$ satisfies the doubling condition, applying Lemma 3.4 and the conclusion of Theorem 1.2 under the condition (c), we get

$$
\left\|T_{\Omega, \alpha} f\right\|_{q, u^{q}} \leq C\|f\|_{p, v^{p}} .
$$

This is the conclusion of Theorem 1.1 for the case (a).

THE PROOF OF (1.7) FOR THE CONDITION (b) IN THEOREM 1.1. Since the fractional integral operator $T_{\Omega, \alpha}$ is a linear operator, we denote $T^{*}:=\left(T_{\Omega, \alpha}\right)^{*}$ as the adjoint operator of $T_{\Omega, \alpha}$. Then $\left(T_{\Omega, \alpha}\right)^{*}=T_{\Omega^{*}, \alpha}$, where $\Omega^{*}(x)=\overline{\Omega(-x)}$. Clearly, $\Omega^{*}$ satisfies the same conditions as $\Omega$. We have

$$
\left\|T_{\Omega, \alpha} f\right\|_{q, u^{q}}=\sup _{g}\left|\int_{\mathbb{R}^{n}} T_{\Omega, \alpha} f(x) g(x) d x\right|,
$$

where the supremum is taken over all $g$ with $\|g\|_{q^{\prime}, u^{-q^{\prime}}} \leq 1$. On the other hand,

$$
\int_{\mathbb{R}^{n}} T_{\Omega, \alpha} f(x) g(x) d x=\int_{\mathbb{R}^{n}} f(x) T^{*} g(x) d x .
$$

Thus, by Hölder's inequality, we get

$$
\left\|T_{\Omega, \alpha} f\right\|_{q, u^{q}}=\sup _{g}\left|\int_{\mathbb{R}^{n}} T_{\Omega, \alpha} f(x) g(x) d x\right| \leq\|f\|_{p, v^{p}} \sup _{g}\left\|T^{*} g\right\|_{p^{\prime}, v^{-p^{\prime}}} .
$$

From condition (b) of Theorem 1.1, we see that $1 / p^{\prime}=1 / q^{\prime}-\alpha / n$ and $s^{\prime}<q^{\prime}<n / \alpha$. Since $\left(v^{-s^{\prime}}, u^{-s^{\prime}}\right) \in A^{*}\left(q^{\prime} / s^{\prime}, p^{\prime} / s^{\prime}\right)$ and $v(x)^{-s^{\prime}}, v(x)^{-s^{\prime}} \in A\left(q^{\prime} / s^{\prime}, p^{\prime} / s^{\prime}\right)$, using the conclusion of Theorem 1.1 for the case (a), we get

$$
\left\|T^{*} g\right\|_{p^{\prime}, v^{-p^{\prime}}} \leq C\|g\|_{q^{\prime}, u^{-q^{\prime}}}
$$

Therefore,

$$
\left\|T_{\Omega, \alpha} f\right\|_{q, u^{q}} \leq\|f\|_{p, v^{p}} \cdot \sup _{g}\left\|T^{*} g\right\|_{p^{\prime}, v^{-p^{\prime}}} \leq C\|f\|_{p, v^{p}} .
$$

This is the inequality (1.7) in Theorem 1.1.

THE PROOF OF (1.8) FOR THE CONDITION (d) IN THEOREM 1.2. Finally, we show how to obtain the weighted inequality (1.8) for the case (d) in Theorem 1.2. Note that the conclusions of Theorem 1.1 hold also for $T_{|\Omega|, \alpha}(|f|)$, hence inequality (1.8) for the case (d) is a direct result of the following lemma and the conclusion of Theorem 1.1 for the case (b).

LEMMA 3.5 (see [2]). Let $0<\alpha<n, \Omega \in L^{1}\left(S^{n-1}\right)$. Then we have

$$
M_{\Omega, \alpha} f(x) \leq T_{|\Omega|, \alpha}(|f|)(x), \quad x \in \mathbb{R}^{n} .
$$


ACKNOWLEDGEMENT. Yong Ding was partially supported by NSF of China (Grant No. 19971010).

\section{REFERENCES}

[1] S. Chanillo, D. K. Watson, and R. L. Wheeden, Some integral and maximal operators related to starlike sets, Studia Math. 107 (1993), no. 3, 223-255. MR 94j:42027. Zbl 809.42008.

[2] Y. Ding, Weak type bounds for a class of rough operators with power weights, Proc. Amer. Math. Soc. 125 (1997), no. 10, 2939-2942. MR 97m:42013. Zbl 887.42008.

[3] Y. Ding and S. Lu, Weighted norm inequalities for fractional integral operators with rough kernel, Canad. J. Math. 50 (1998), no. 1, 29-39. MR 99c:42028. Zbl 905.42010.

[4] J. García-Cuerva and J. L. Rubio de Francia, Weighted Norm Inequalities and Related Topics, North-Holland Mathematics Studies, vol. 116. Notas de Matematica, 104, NorthHolland Publishing Co., New York, 1985. MR 87d:42023. Zbl 578.46046.

[5] B. Muckenhoupt, Weighted norm inequalities for the Hardy maximal function, Trans. Amer. Math. Soc. 165 (1972), 207-226. MR 45\#2461. Zbl 236.26016.

[6] B. Muckenhoupt and R. L. Wheeden, Weighted norm inequalities for singular and fractional integrals, Trans. Amer. Math. Soc. 161 (1971), 249-258. MR 44\#3155. Zbl 226.44007.

[7] C. J. Neugebauer, Inserting $A_{p}$-weights, Proc. Amer. Math. Soc. 87 (1983), no. 4, 644-648. MR 84d:42026. Zbl 521.42019.

[8] E. T. Sawyer, A characterization of a two-weight norm inequality for maximal operators, Studia Math. 75 (1982), no. 1, 1-11. MR 84i:42032. Zbl 508.42023.

[9] R. L. Wheeden, A characterization of some weighted norm inequalities for the fractional maximal function, Studia Math. 107 (1993), no. 3, 257-272. MR 94m:42044. Zbl 809.42009.

Yong Ding: DePartment of MATHematics, Beijing Normal UNiversity, BeiJing 100875, CHINA

E-mail address: dingy@bnu.edu.cn

Chin-Cheng Lin: Department of Mathematics, National Central University, ChungLI, 320, TAIWAN

E-mail address: c1 in@math.ncu.edu. tw 


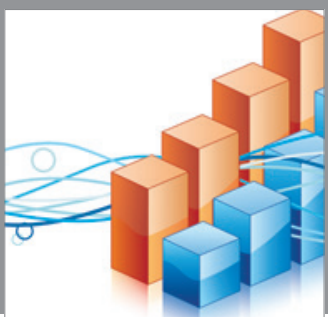

Advances in

Operations Research

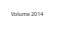

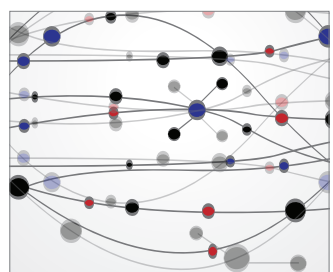

\section{The Scientific} World Journal
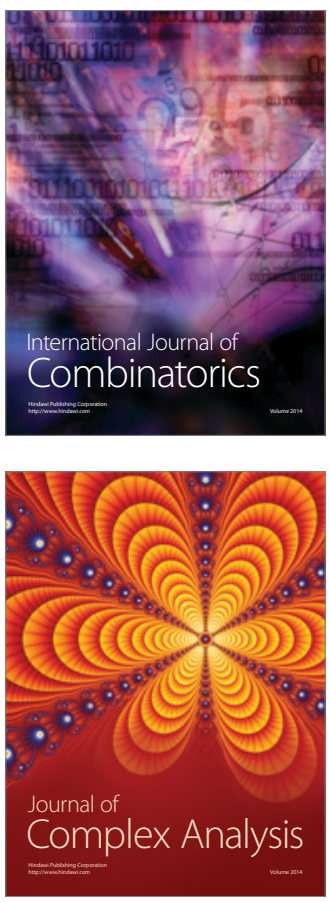

International Journal of

Mathematics and

Mathematical

Sciences
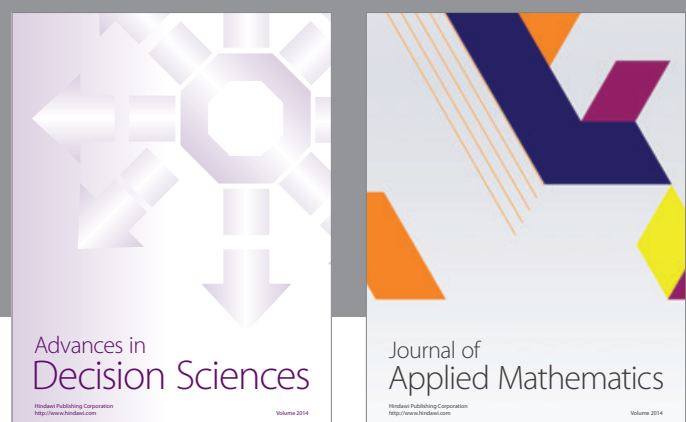

Journal of

Applied Mathematics
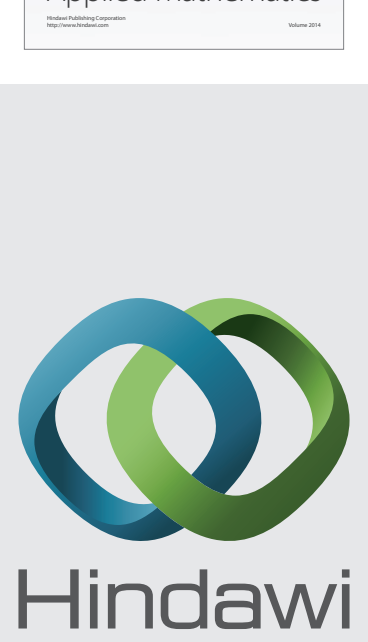

Submit your manuscripts at http://www.hindawi.com
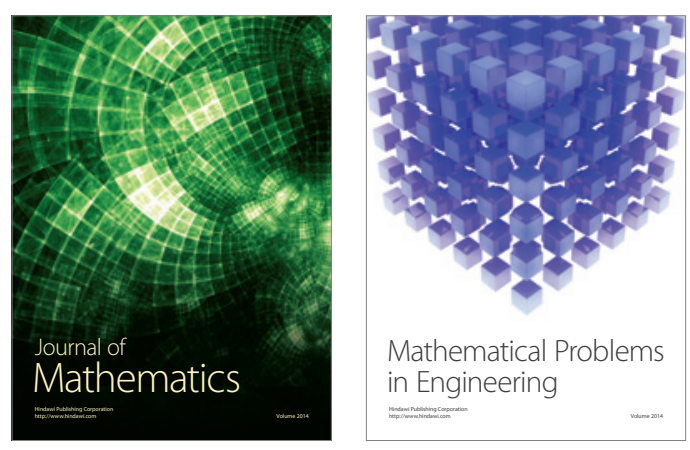

Mathematical Problems in Engineering
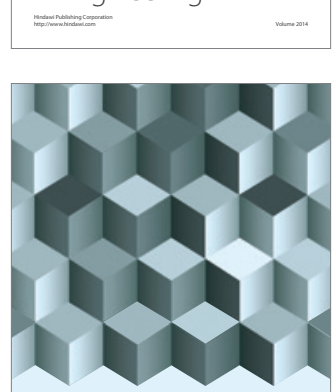

Journal of

Function Spaces
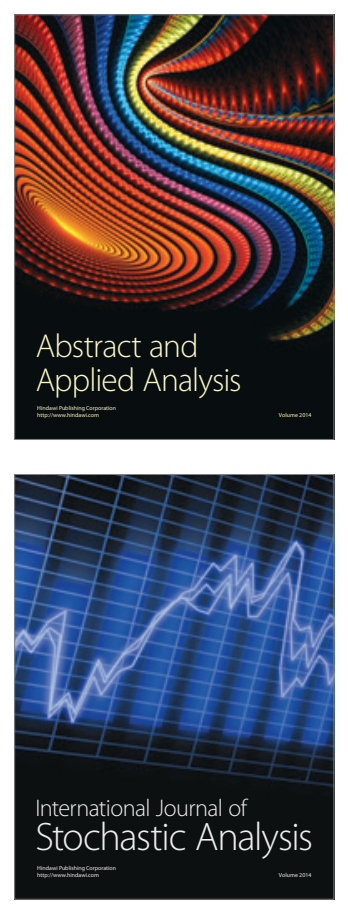

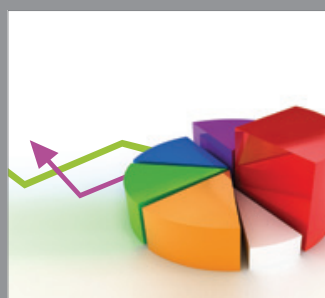

ournal of

Probability and Statistics

Promensencen
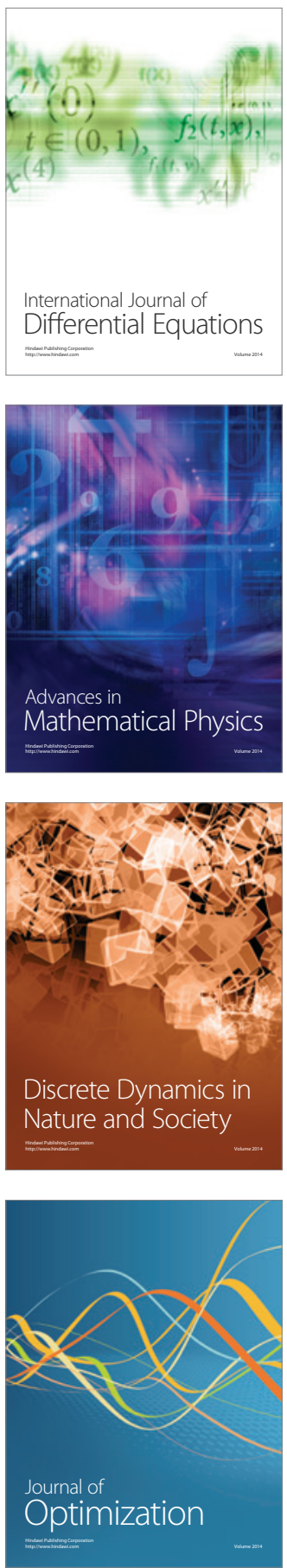